\title{
Progress towards Bell-type polarization experiment with thermal neutrons
}

\author{
M. Iannuzzi, ${ }^{1}$ R. Messi, ${ }^{1,2}$ D. Moricciani, ${ }^{2,}{ }^{*}$ A. Orecchini, ${ }^{3,4}$ and F. Sacchetti ${ }^{3}$ \\ ${ }^{1}$ Dipartimento di Fisica, Università di Roma “Tor Vergata," I-00133 Roma, Italy \\ ${ }^{2}$ INFN Sezione “Roma Tor Vergata," I-00133 Roma, Italy \\ ${ }^{3}$ Dipartimento di Fisica e Geologia, Università di Perugia, I-06123 Perugia, Italy \\ ${ }^{4}$ Institut Laue-Langevin, F-38042 Grenoble, France \\ (Received 5 September 2014; published 25 February 2015)
}

\begin{abstract}
Experimental tests of Bell-type inequalities distinguishing between quantum mechanics and local realistic theories remain of considerable interest if performed on massive particles, for which no conclusive result has yet been obtained. Only two-particle experiments may specifically test the concept of spatial nonlocality in quantum theory, whereas single-particle experiments may generally test the concept of quantum noncontextuality. Here we have performed the first Bell-type experiment with a beam of thermal-neutron pairs in the singlet state of spin, as originally suggested by J. S. Bell. These measurements confirm the quantum-theoretical predictions, in agreement with the results of the well-known polarization experiments carried out on optical photons years ago.
\end{abstract}

PACS number(s): 03.65.Ud, 03.65.Ta

Since the starting point of Bell's theorem on nonlocality of the quantum theory in 1965 [1], theoretical speculations and search for experimental verifications of various inequalities derived from the theorem have been pursued over the years. Most of the performed experiments were based on correlations between the polarization of pairs of photons, in particular, high-energy photons produced by positronium annihilation [2], or optical photons emitted by atomic cascade [3,4] or by parametric down-conversion [5]. One test was also done on massive particles to measure the spin correlation in proton pairs prepared in low-energy proton-proton scattering [6]. These early experiments on positronium annihilation and proton-proton scattering did not give conclusive results, mainly because of the lack of efficient linear polarization filters both for protons and high-energy photons, and because of other difficulties related to the apparatus. One more recent and complex test performed on massive particles, pairs of entangled ${ }^{9} \mathrm{Be}^{+}$ions, appears as conceptually questionable because the manipulations of the two particles of a pair were not done individually and independently of each other, as would be required to verify spatial nonlocality [7]. The extensive tests working with atomic cascades producing pairs of optical photons have been the most successful (together with those completed afterward by using parametric down-conversion), mainly because of the availability of very efficient polarization filters and an efficient technique for the manipulation of visible light beams. The experimental limitations of such experiments are mainly related to the relatively low quantum efficiency of the detectors and the difficulty to select and detect both members of a photon pair, because the variable recoil momentum of the emitting atom diminishes their direction correlation. However, with a supplementary assumption justifying that the detected photon pairs constitute a statistically representative sample of the whole ensemble of photons emitted from the source, these experiments confirm the predictions of the quantum theory with great accuracy. A detailed and comprehensive analysis of the three classes of test experiments performed on Bell's

*Corresponding author: dario.moricciani@ roma2.infn.it theorem (e.g., atomic cascades, proton-proton scattering, positronium decay), is given in [8].

The above considerations, and particularly the limits of the experiments with massive particles, have motivated us to perform a Bell-type experiment working with a collimated beam of thermal neutrons prepared in such a way that, within the experimental limitations of the measurement, neutron pairs in the singlet state of spin were identified by the detecting system. We recall that an experimental test on such a physical system is particularly interesting since Bell's inequality is violated by a large amount in the singlet state. We also recall that beautiful interferometric measurements have been done with single-neutron beams, testing correlations between two degrees of freedom of the single particle [9-11]. However, such experiments are not equivalent to those performed with twoneutron beams, which refer to the issue of spatial nonlocality for two separated particles. This latter concept is meaningless for a single particle, and the interferometric experiments have rather tested the general concept of quantum noncontextuality.

Following the results of the previous measurements on the fermion antibunching [12,13], the present measurements have been carried out at the Institute Laue Langevin (Grenoble, France) by using the primary spectrometer of instrument IN10, which produces a monochromatic beam of thermal neutrons from an almost perfect $\mathrm{Si}(111)$ single crystal in the almost perfect backscattering configuration. The neutron intensity at the test position was $2 \times 10^{3} \mathrm{n} / \mathrm{s}$ on a beam size of $3 \times 4 \mathrm{~cm}^{2}$. The energy spread cannot be measured directly but it can be estimated from the monochromator geometry to be $\Delta E<$ $0.02 \mu \mathrm{eV}$, corresponding to a coherence time $\tau_{c}=\hbar / \Delta E>$ $20 \mathrm{~ns}$ at $6.27 \AA$. The distance of the multianode detector from the monochromator was $\sim 12 \mathrm{~m}$ and $\sim 2 \mathrm{~m}$ from the collimator exit; the whole equipment was within the shielding of IN10.

The extraction of a beam of correlated particles in the singlet state can be described as follows. The nature of the emission of thermal neutrons in the source (the neutron moderator of ILL) is Poissonian, so that there is a small but finite probability of having two neutrons within the detection time of our apparatus. The beam emitted from the source is monochromatized by reflection on a quasiperfect Si crystal. The beam being spin unpolarized, it is equally likely that 
a neutron pair emitted within the coherence time $\tau_{c}$ of the monochromator will either occur in one of the three triplet states or in the singlet state, i.e., the triplet states will occur $3 / 4$ of the time. In a gas of fermions there is the tendency for particles of the same spin to avoid each other, a tendency arising from the exchange antisymmetry of the wave function. More specifically, the two neutrons of a pair emitted in the triplet state and traveling along the long collimators $(\sim 10 \mathrm{~m})$ from the monochromator to the polarizers are in the same spatial mode (antisymmetrical spatial wave function), so that they avoid each other scattering in directions different from the one of the emerging collimated beam, and they will not reach the detectors. The neutrons of a pair in the singlet state do not avoid each other traveling along the collimators; they will pass through the two separated polarizers, and after them, are distinguishable particles that will be detected without exhibiting interference effects. Consequently, within a time interval of the order of $\tau_{c}$, only neutron pairs emitted from the source in the singlet state can be detected in our apparatus. This is the physical effect already measured in [12,13], and applied in the present experiment.

The conceptual scheme of the experiment was similar to the one described in [13], with the addition of two polarizers (1 and 2 in Fig. 1), each one mounted along two slightly different neutron paths from the beam output window. Both polarizers consisted of a disk, $3.8 \mathrm{~cm}$ diameter and $2.6 \mathrm{~cm}$ thick, made of $\mathrm{Fe}_{3} \mathrm{Al}$ intermetallic compound and mounted between the poles of a $0.2 \mathrm{~T}$ permanent magnet. The $\mathrm{Fe}_{3} \mathrm{Al}$ intermetallic compound was chosen because the first Bragg edge, corresponding to the (111) reflection of its $\mathrm{DO}_{3}$ structure, is at $\lambda=6.67 \AA$, while the second Bragg edge due to the (200) reflection is at $\lambda=5.78 \AA$. So, at the working neutron wavelength of $6.67 \AA$ the transmission of the disk is due only to the capture cross section of $\mathrm{Fe}$ and to the (111) Bragg scattering. If a polarizer is magnetically saturated, the cross section is large when the $S_{z}$ component of the neutron

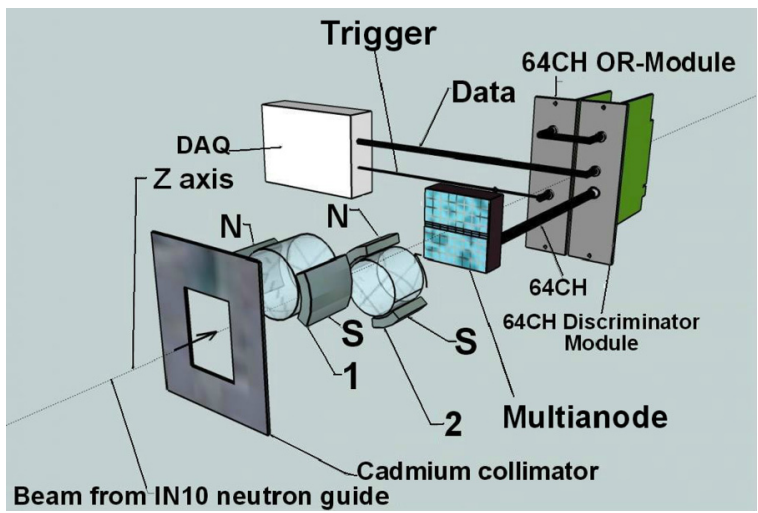

FIG. 1. (Color online) Scheme of the experiment. Distances along the $z$ direction are not on scale. Sizes of the neutron guide and collimating window are, respectively, $3 \times 3 \mathrm{~cm}^{2}$ and $3 \times 4 \mathrm{~cm}^{2}$. 64CH: 64 channels from the multianode pixels; DAQ: data acquisition system; OR-module is a logical circuit triggering DAQ on receiving a signal from whatever channel of the $64 \mathrm{CH}$ discriminator module. The polarizers are separated vertically, fronting the two halves of the detectors; erroneous visual impression may arise from the drawing perspective. spin is parallel to the magnetization, and it is small when $S_{z}$ is antiparallel [14]. With our $0.2 \mathrm{~T}$ magnet the measured transmission coefficients were $\varepsilon^{\downarrow}=22.1 \%$ and $\varepsilon^{\uparrow}=8.4 \%$. In the present apparatus, polarizer 2 was mounted on a motorized rotation stage and could be rotated with respect to polarizer 1 mounted within a fixed horizontal field. The relative angle $\vartheta$ of their polarization axes was variable in the range from $0^{\circ}$ to $360^{\circ} \pm 0.1^{\circ}$. Since the polarizers can be considered as state preparation devices, their position along the neutron path was not important, provided that the two neutron paths were identical as it was in our apparatus.

As in [13], the position-sensitive detector was a Hamamatsu H8500 multianode photomultiplier having $8 \times 8$ anode pixels with a pixel size of $5.8 \times 5.8 \mathrm{~mm}^{2}$. The photomultiplier was coupled to a $0.15 \mathrm{~mm}$ thick lithium glass scintillator $\left({ }^{6} \mathrm{Li}\right.$ $98 \%$ enriched) directly coupled to the anode window with a thin layer of optical grease. The scintillator was divided into two different parts with a separation distance of $\simeq 0.4$ $\mathrm{mm}$, so that each part of the scintillator was looking at the neutrons passing through either polarizer 1 or polarizer 2 , and there was no cross-talk between the two detecting areas due to the neutrons impinging on the separation area. Specific pixel groupings could be defined during the off-line analysis to optimize the view of the upper and lower parts of the neutron beam, so that each of the two detectors, $D_{1}$ and $D_{2}$, recorded the neutrons passing through one specific polarizer. Generally each detector was formed of a row of 4-6 pixels with total area $\leqslant 3.0 \times 0.5 \mathrm{~cm}^{2}$, chosen so as they were illuminated only by those neutrons that, filtered respectively by polarizer 1 and polarizer 2, could not be intercepted by the Fe magnetic poles of polarizer 2 at any angle $\vartheta$. The neutron arrival time at each pixel was measured by using an internal $40 \mathrm{MHz}$ clock which provided $25 \mathrm{~ns}$ time resolution, shorter than both the light decay time of the lithium glass scintillator ( $\simeq 250 \mathrm{~ns}$ ) and the neutron total traveling time through the scintillating sheet $(\simeq 240 \mathrm{~ns})$. The data acquisition system monitored events during repeated cycles of $10 \mathrm{~s}$ each, with an effective duty cycle of about $99.90 \%$, where the dead time due to the VME readout cycle was of the order of $10 \mathrm{~ms}$.

In order to determine the spin correlation of two neutrons, a time stamp was attributed to the neutron signals from the two detectors, so that the neutron pair was time correlated when two neutrons arrived at the two detectors $D_{1}$ and $D_{2}$ with time stamp $t_{1}=$ start and $t_{2}=$ stop. In the off-line analysis we could study the coincidence rate as a function of $\delta=t_{2}-t_{1}$, or in other words, as a function of the virtual spatial separation $v_{\text {ther }} \delta$ along the propagation direction $z$. In $[12,13]$ it is also shown that, thanks to the specific characteristics of the IN10 primary spectrometers, there is a good neutron correlation when the two detectors $D_{1}$ and $D_{2}$ are separated in the transverse direction up to a couple of centimeters. The issue of the existence or amplitude of macroscopic lateral coherence and its possible effects on coincidence measurements is controversial. For this reason we prefer to limit ourselves to report our measured value. We have no conclusive interpretation of this result, and we feel that this effect requires further experimental investigation.

According to the above description, the experimental results that we are presenting provide the number of neutrons 
monitored by $D_{2}$ at a time $t_{2}$ delayed by $\delta$ with respect to time $t_{1}$ when the first neutron is detected at $D_{1}$. It is interesting to obtain a calculation of this number from the convolution of the predicted neutron-correlation function $c(\vartheta, \delta)$, and the response function of the detecting system $w(\delta)$.

The function $w(\delta)=W \exp \left[-\delta / t_{w}\right]$ describes the time broadening of the detecting system due to the scintillator thickness and to the decay time of the emitted light. Actually, from the capture probability profile of the neutron along the scintillator thickness, the average capture time is about $t_{c}=80 \mathrm{~ns}$ with an additional broadening from the light decay curve of the order of $t_{d}=250 \mathrm{~ns}$. By considering that both effects can be roughly described by an exponential function, the total decay time is expected to be $t_{w}=\left(1 / t_{c}+\right.$ $\left.1 / t_{d}\right)^{-1}=60 \mathrm{~ns}$. The choice of the Gaussian form of $c(\vartheta, \delta)=$ $1-\alpha(\vartheta) \exp \left[-\delta^{2} /\left(2 \tau_{c}^{2}\right)\right]$ was dictated first by the need of describing reasonably (similarly to what was done in [12]) the antibunching effect which produces, for $\delta=0$ and for any value of $\vartheta$, a $3 / 4$ depression of the coincidence counts with respect to the random coincidences occurring for $\delta \gg \tau_{c}$; and second by the need to add also the simultaneous depression produced by the presence of the polarizers on the neutron pairs in the singlet state [see prediction (7), recalled later in the text]. The value of the coefficient $\alpha(\vartheta)$ must be $3 / 4+1 / 4=1$ for $\vartheta=0^{\circ}$. From the convolution $[w * c] c\left(t^{\prime}\right)$ of $w$ and $c$, the number of coincidences is then given by

$$
C(\vartheta, \delta)=\frac{C(\vartheta, \infty)}{\Delta} \int_{\delta}^{\delta+\Delta}[w * c]\left(t^{\prime}\right) d t^{\prime}, \quad \delta>0
$$

where $C(\vartheta, \delta)$ is the number of experimental coincidences as a function of $\delta, C(\vartheta, \infty)$ is the number of experimental coincidences for $\delta \gg \tau_{c}, \Delta$ is the coincidence time window of the order of $\tau_{c}$, and $W=1$. In Fig. 2 the experimental results are compared with the prediction of Eq. (1). Tests taking $\alpha(0)$, $t_{w}$, and $\tau_{c}$ as free parameters confirmed our estimates, and we found $\alpha(0)=1 \pm 0.1, t_{w}=60 \pm 9 \mathrm{~ns}$, and $\tau_{c}=78 \pm 10 \mathrm{~ns}$, in agreement with the previous estimates [13].

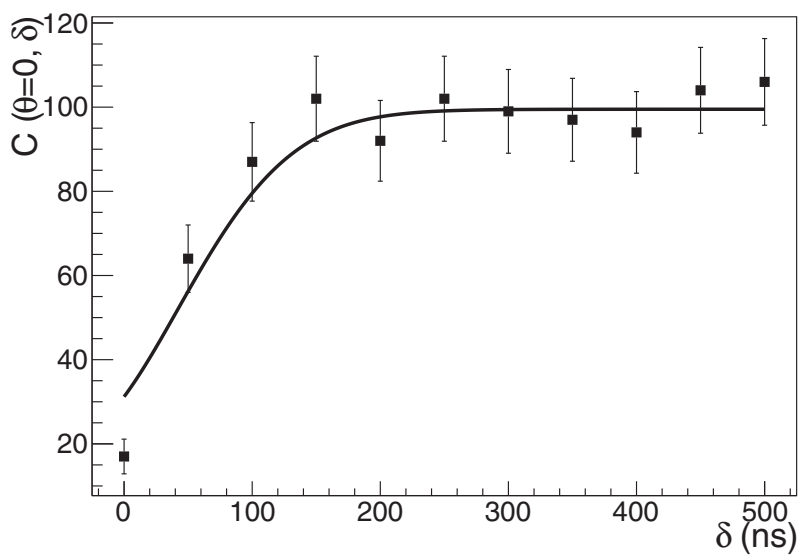

FIG. 2. $C(\vartheta=0, \delta)$ is shown as a function of the neutron time delay $\delta=t_{2}-t_{1}$ along the longitudinal $z$ direction; full square: experimental points. Two 4-pixel rows with relative separation 0.4 $\mathrm{cm}, \Delta=150 \mathrm{~ns}$.

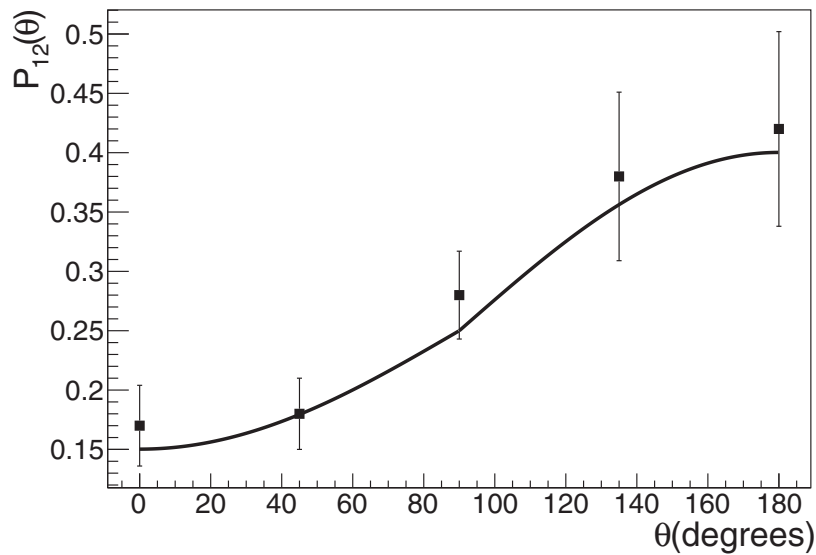

FIG. 3. $P_{12}(\vartheta)$ as a function of the angle $\vartheta$ between the polarization axes. Two 4-pixel rows with relative separation $0.4 \mathrm{~cm}$, and $\Delta=150$ ns. Full line: quantum prediction [Eq. (6)].

We can now define the function:

$$
P_{12}(\vartheta)=\frac{C(\vartheta, 0)}{C(\vartheta, \infty)},
$$

which is independent of all possible fluctuations of the beam intensity and other sources of instrumental uncertainties. It represents the experimental value of the probability of detecting the simultaneous occurrence, after transmission through the linear polarization analyzers, of the two neutrons of a pair emitted in the singlet state, with respect to all four states of the physical system. The experimental results obtained for $P_{12}(\vartheta)$ at five different values of $\vartheta$ are shown in Fig. 3. The acquisition time of each experimental point was in the range $18-24 \mathrm{~h}$.

In order to obtain the quantum-theoretical prediction for the outcome of the experiment, let us first calculate the probability $P_{12}(\vartheta)$ for perfect analyzers that transmit only neutrons with spin antiparallel to the magnetic field. During a given time interval, the source emits $N$ neutron pairs. For $N$ large enough, we define $N_{i} / N=c_{i}, N_{i}$ being the number of counts at detector $i$; and we also recall that $N_{i}=N / 2$ is the number of neutrons transmitted through perfect polarizer $i$ when $\delta=t_{2}-t_{1} \gg \tau_{c}$. Since the fraction of the neutron pairs emitted in the singlet state and recorded after the polarizers at short time delays is $1 / 4$ of all the pairs, we obtain

$$
P_{12}(\vartheta)=\frac{1}{4}\left\langle\psi_{s}\left|Q_{1}^{\downarrow} Q_{2}^{\downarrow}\right| \psi_{s}\right\rangle=\frac{1}{4} \frac{[1-\cos (\vartheta)]}{4},
$$

where $\left|\psi_{s}\right\rangle$ is the singlet wave function and $Q_{i}^{\downarrow}$ is the operator which projects the spin of particle $(i)$ on the direction of magnetic field with value $-\hbar / 2$. Yet a more realistic analysis, taking account of the limitations of the apparatus, can be adopted for comparison with the real experiment. We can adapt to result (3) the analysis adopted by Clauser, Horne, and Shimony (CHS) $[8,15]$ for pairs of optical photons with parallel linear polarization. They derive the following prediction: $P_{12}(\vartheta)=c_{1} c_{2}\left[\varepsilon_{1}^{+} \varepsilon_{2}^{+}+\varepsilon_{1}^{-} \varepsilon_{2}^{-} \cos (2 \vartheta)\right] / 4$ and $P_{12}(\infty, \infty)=$ $c_{1} c_{2}$ with $\varepsilon^{+}=\varepsilon_{i}^{M}+\varepsilon_{i}^{m}, \varepsilon^{-}=\varepsilon_{i}^{M}-\varepsilon_{i}^{m}$, where $\varepsilon_{i}^{M}$ and $\varepsilon_{i}^{m}$ are, respectively, the maximum and minimum transmittance of polarizer $i$. The symbol $\infty$ defines the absence of a polarizer. 
By adapting their approach, particularly the one in [8], to our system of thermal neutrons, we have not performed a direct mathematical derivation of their result, because the significative differences to be considered between the two cases have dictated to introduce changes in their calculation. First we recall that a linearly polarized photon propagating along the $z$ axis has polarization direction in the $x-y$ plane; in contrast, a neutron propagating along the $z$ axis has spin components different from 0 only in one $x-y$ semiplane. We also recall that the optical linear polarizers have the maximum transmittance in direction perpendicular to that of minimum transmittance, while thermal neutron polarizers have maximum and minimum transmittance in antiparallel directions. Second, only coincidences occurring within a time interval $\delta \leqslant \tau_{c}$ are considered in our analysis, i.e., coincidences due to pairs of neutrons in the singlet state.

We must now remark that a neutron polarizer like ours is a linear polarizer with oriented polarization axis, so that two of such perfect polarizers $\left(\varepsilon_{1}^{\downarrow}=1\right.$ and $\left.\varepsilon_{2}^{\uparrow}=0\right)$ with parallel orientation of their axes cannot transmit two correlated neutrons in the singlet state (antiparallel spins), and coincidences may be observed only in the angular range $90^{\circ} \leqslant \vartheta \leqslant 180^{\circ}$. Consequently, the ideal result (3) can only be confronted with Eq. (6), obtained later in the text, in this angular range. However, with real polarizers $\left(\varepsilon_{1}^{\downarrow}=0.221\right.$ and $\varepsilon_{2}^{\uparrow}=0.084$ in our experiment) with oriented polarization axis, coincidence counts may also occur for $0^{\circ} \leqslant \vartheta \leqslant 90^{\circ}$, but still the probability of their occurrence cannot be confronted with prediction (3).

As a consequence of such differences between optical photons and neutrons, in the above CHS equation $\varepsilon_{i}^{+}$and $\varepsilon_{i}^{-}$ have become, respectively, $\varepsilon_{i}^{\downarrow}$ and $\varepsilon_{i}^{\uparrow}$ in the term proportional to $\cos (\vartheta)$. In addition, note that the neutron correlation function $\sin ^{2}(\vartheta / 2) / 2$ [see Eq. (7)] substitutes the photon correlation function $\cos ^{2}(\vartheta) / 2$; the symbol $\infty$ does not denote the absence of a polarizer, it denotes the absence of correlation between to two spins of a pair for $\delta \gg \tau_{c}$ instead; the beam of neutrons in the singlet state is the fraction $1 / 4$ of the whole ensemble emitted from the monochromator.

Following the above considerations, we obtain for $0^{\circ} \leqslant$ $\vartheta \leqslant 90^{\circ}$,

$$
\begin{aligned}
C(\vartheta, 0) & =\frac{c_{1} c_{2}}{4}\left[\frac{\varepsilon_{1}^{t} \varepsilon_{2}^{t}-\left(\varepsilon_{1}^{\downarrow} \varepsilon_{2}^{\uparrow}+\varepsilon_{1}^{\uparrow} \varepsilon_{2}^{\downarrow}\right) \cos (\vartheta)}{4}\right], \\
C(\vartheta, \infty) & =\frac{c_{1} c_{2} \varepsilon_{1}^{t} \varepsilon_{2}^{t}}{4},
\end{aligned}
$$

and for $90^{\circ} \leqslant \vartheta \leqslant 180^{\circ}$,

$$
\begin{aligned}
C(\vartheta, 0) & =\frac{c_{1} c_{2}}{4}\left[\frac{\varepsilon_{1}^{t} \varepsilon_{2}^{t}-\left(\varepsilon_{1}^{\uparrow} \varepsilon_{2}^{\uparrow}+\varepsilon_{1}^{\downarrow} \varepsilon_{2}^{\downarrow}\right) \cos (\vartheta)}{4}\right], \\
C(\vartheta, \infty) & =\frac{c_{1} c_{2} \varepsilon_{1}^{t} \varepsilon_{2}^{t}}{4}
\end{aligned}
$$

where $\varepsilon_{i}^{t}=\varepsilon_{i}^{\uparrow}+\varepsilon_{i}^{\downarrow}$. Following the definition of Eq. (2) and assuming identical polarizers $\left(\varepsilon_{1}^{\downarrow} \varepsilon_{2}^{\uparrow}=\varepsilon_{1}^{\uparrow} \varepsilon_{2}^{\downarrow}\right)$, we derive

$P_{12}(\vartheta)=\frac{1}{4}\left[1-\frac{2 \varepsilon_{1}^{\downarrow} \varepsilon_{2}^{\uparrow}}{\varepsilon_{1}^{t} \varepsilon_{2}^{t}} \cos (\vartheta)\right]$ for $0^{\circ} \leqslant \vartheta \leqslant 90^{\circ} \quad$ and
$P_{12}(\vartheta)=\frac{1}{4}\left[1-\frac{\varepsilon_{1}^{\uparrow} \varepsilon_{2}^{\uparrow}+\varepsilon_{1}^{\downarrow} \varepsilon_{2}^{\downarrow}}{\varepsilon_{1}^{t} \varepsilon_{2}^{t}} \cos (\vartheta)\right]$ for $90^{\circ} \leqslant \vartheta \leqslant 180^{\circ}$.

The results obtained by using Eq. (6) are shown in Fig. 3 (full line) as a function of $\vartheta$. Clearly the theoretical prediction of the quantum theory is in agreement with the experimental points, within the errors of the present experiment.

Recall also that for an analogous experiment which might be performed with ideal instrumentation and with a source emitting only neutron pairs in the singlet state, the quantum prediction substituting Eq. (6) is

$$
P_{12}^{\text {ideal }}(\vartheta)=\left\langle\psi_{s}\left|Q_{1}^{\downarrow} Q_{2}^{\downarrow}\right| \psi_{s}\right\rangle=\frac{[1-\cos (\vartheta)]}{4},
$$

and that this simple sinusoidal form of the ideal prediction (as other simple sinusoidal forms of quantum predictions, in other cases) is the sole origin for the violations of the $\mathrm{CH}$ inequality [16].

Let us summarize the most significant experimental features of the present measurement: (1) from the highly monochromatic and collimated beam of thermal neutrons produced at the IN10 beam of the ILL, by utilizing the antibunching effect of the neutrons in the triplet states, the two members of a pair in the singlet state could be detected at small time separation as a function of the angle $\vartheta$ between the two polarizers; (2) the effects on the data analysis due to the presence of undesirable and uncertain instrumental origin were greatly reduced by measuring $P_{12}(\vartheta)$ from the ratio of the coincidence rate at small time separation to the coincidence rate at large time separation $\left(t_{2}-t_{1} \gg \tau_{c}\right)$.

The measurement seems adequate to confirm the quantumtheoretical prediction (6) which takes account of the real limitations of the present experiment, and, by extrapolation, the theoretical prediction (7) for an ideal experiment performed with perfect instrumentation.

We wish to remark explicitly that the efficiency of our polarizers was insufficient for a decisive confrontation of the experimental data with the Clauser-Horne $(\mathrm{CH})$ inequality, confrontation which requires very high efficiency of all of the components of the apparatus. We also note that such limitation prevents one from performing loophole-free measurements. On the other hand, we consider our result as a helpful contribution to this issue. Actually, the confirmation of quantum prediction (6) could but be reinforced by experiments performed with greater efficiency and statistical precision, as was shown in the optical polarization experiments, and also commented by Bell [17]: “. . . it is hard for me to believe that quantum mechanics works so nicely for inefficient practical set-up and is yet going to fail badly when sufficient refinements are made...." Therefore, our results seem adequate to verify the predictions of the quantum theory for massive particles in the spin singlet state and, although by extrapolation from the real measurement, to confirm violations of the $\mathrm{CH}$ inequality.

We are grateful to P. Facchi and S. Pascazio for enlightening discussions and we acknowledge the Institut Laue Langevin of Grenoble for the beam time, which enabled the realization of the experiment. 
[1] J. S. Bell, Physics (NY) 1, 195 (1964).

[2] M. Bruno, M. D'Agostino, and C. Maroni, Nuovo Cimento B 40, 143 (1977), and references therein of preceding works with positronium annihilation.

[3] S. J. Freeman and J. F. Clauser, Phys. Rev. Lett. 28, 938 (1972).

[4] A. Aspect, P. Grangier, and G. Roger, Phys. Rev. Lett. 49, 91 (1982), and references therein.

[5] G. Weihs, T. Jennewein, C. Simon, H. Weinfurter, and A. Zeilinger, Phys. Rev. Lett. 81, 5039 (1998), and references therein. This experiment has definitely confirmed that violations of Bell-type inequalities occur also at spacelike separation of the observations.

[6] M. Lamehi-Rachti and W. Mittig, Phys. Rev. D 14, 2543 (1976).

[7] M. A. Rowe, D. Kielpinski, V. Meyer, C. A. Sackett, W. M. Itano, C. Monroe, and D. J. Wineland, Nature (London) 409, 791 (2001).

[8] J. F. Clauser and A. Shimony, Rep. Prog. Phys. 41, 1881 (1978).
[9] Y. Hasegawa, R. Loidl, G. Badurek, M. Baron and H. Rauch, Nature (London) 425, 45 (2003), and references therein.

[10] Y. Hasegawa, R. Loidl, G. Badurek, S. Filipp, J. Klepp, and H. Rauch, Phys. Rev. A 76, 052108 (2007).

[11] H. Bartosik, J. Klepp, C. Schmitzer, S. Sponar, A. Cabello, H. Rauch, and Y. Hasegawa, Phys. Rev. Lett. 103, 040403 (2009).

[12] M. Iannuzzi, A. Orecchini, F. Sacchetti, P. Facchi, and S. Pascazio, Phys. Rev. Lett. 96, 080402 (2006).

[13] M. Iannuzzi, R. Messi, D. Moricciani, A. Orecchini, F. Sacchetti, P. Facchi, and S. Pascazio, Phys. Rev. A 84, 015601 (2011).

[14] S. J. Pickart and R. Nathans, Phys. Rev. 123, 1163 (1961).

[15] J. F. Clauser and M. A. Horne, Phys. Rev. D 10, 526 (1974). See Eq. (14).

[16] For instance, L. E. Ballentine, Quantum Mechanics (World Scientific, Singapore, 1998), Chap. 20, p. 610.

[17] J. S. Bell, Speakable and Unspeakable in Quantum Mechanics (Cambridge University Press, Cambridge, 1987), p. 139. 